\title{
Nutritional strategies for the rehabilitation of COVID-19 patients
}

\author{
Domenico Azzolino $\mathbb{1}^{1} \cdot$ Pier Carmine Passarelli ${ }^{2} \cdot$ Antonio $\mathrm{D}^{\prime}$ Addona $^{2} \cdot$ Matteo Cesari $^{1,3}$
}

Received: 6 June 2020 / Accepted: 26 October 2020 / Published online: 9 November 2020

(c) Springer Nature Limited 2020

\section{To the Editor:}

We have read with great interest the article by Brugliera et al. [1] reporting the prevalence of dysphagia and malnutrition in COVID-19 patients admitted to the San Raffaele Hospital in Milan (Italy). In their work, authors present a three-step nutritional protocol specifically developed for patients infected by SARS-Cov-2. The study is meritorious especially for its potential implications for healthcare policies as targeting a condition (i.e., malnutrition) that has been severely neglected during this time of pandemic. In particular, Brugliera et al. [1] reported an extremely high prevalence of dysphagia (i.e., $>90 \%$ ) in their COVID-19 patients. Moreover, the vast majority of them (i.e., $>70 \%$ ) presented a moderate-to-marked risk of malnutrition. The protocol the authors implemented at the San Raffaele Hospital has showed very good results, with $43.7 \%$ and $46.8 \%$ of participants showing an increase and stability of the BMI after discharge, respectively.

However, it is important to consider that the assessment of nutritional status via BMI presents some limitations, especially in older people. In fact, the BMI is not a direct measure of adiposity since its numerator (i.e., body weight) includes both fat and fat free mass. With aging, the organism undergoes through the inversion of the ratio

Supplementary Information The online version of this article (https://doi.org/10.1038/s41430-020-00795-0) contains supplementary material, which is available to authorised users.

$\triangle$ Domenico Azzolino

domenico.azzolino@unimi.it

1 Department of Clinical Sciences and Community Health, University of Milan, Milan, Italy

2 Department of Head and Neck, Oral Surgery and Implantology Unit, Institute of Clinical Dentistry, Catholic University of Sacred Hearth, Fondazione Policlinico Universitario Gemelli, Rome, Italy

3 Geriatric Unit, IRCCS Istituti Clinici Scientifici Maugeri, Milan, Italy between muscle mass and fat mass, the so-called condition of sarcopenic obesity, which is characterized by (1) a qualitative worsening of the muscle (due to intra- and interinfiltrates of fat), and (2) the association with negative health-related outcomes [2]. Furthermore, body weight and BMI can be confounded by the presence of ascites and edema in some individuals. Besides of weakening the reliability of these measures (especially in older and complex patients), the excess of fluids also impacts on the results of the bioelectrical impedance analysis.

The impact of COVID-19 on nutritional status cannot be entirely explained by older age and pathologies, as suggested in the article. Functions, age-related physiological modifications, and psycho-social factors also need to be considered as major contributors of malnutrition (Fig. 1) [3].

In particular, advancing age is associated with an increase of circulating levels of pro-inflammatory cytokines, the so-called "inflamm-aging" phenomenon. Interestingly, adipose tissue is today recognized as an endocrine organ producing a variety of pro-inflammatory cytokines and adipokines. This explains why the excess of fat mass is able to generate a vicious cycle worsening the consequences of obesity via a parallel enhancement of the inflammatory cascade [4]. It has been suggested that the SARS-Cov-2 infection can trigger a rapid activation of the innate immune cells. In fact, infected patients tend to present markedly elevated levels of pro-inflammatory cytokines and chemokines [5]. It seems logical that the obesity background of the individual may exponentially increase the inflammatory reaction of the organism to the pathogen, determining the most severe cases in this population.

Another aspect to consider in addition to what presented by Brugliera et al. [1] is that sarcopenia is not limited to lower limbs, but should be recognized as a whole body process, also affecting respiratory, masticatory, and swallowing muscles [6]. Recently, the construct of a "sarcopenic dysphagia", characterized by the concomitant presence of sarcopenia and dysphagia, has been evoked [7]. Consistently, another condition representing an early phase of 


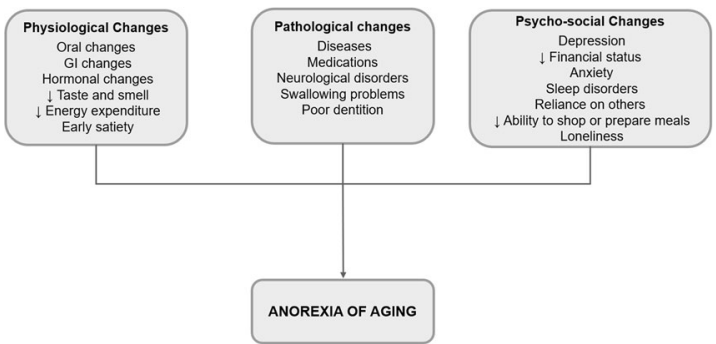

Fig. 1 Major changes occurring with aging. $\uparrow$ increased $\downarrow$ decreased.
Table 1 Assessment procedures for the diagnosis of dysphagia.

\begin{tabular}{ll}
\hline Procedure & Description \\
\hline 1. Screening & $\begin{array}{l}\text { Rapid and simple tests designed to early detect signs and } \\
\text { symptoms of swallowing disorders. }\end{array}$ \\
$\begin{array}{l}\text { 2. Clinical } \\
\text { assessment }\end{array}$ & $\begin{array}{l}\text { Comprehensive and multidimensional assessment of the } \\
\text { dysphagic signs and symptoms of the individual. } \\
\text { 3. Instrumental } \\
\text { assessment }\end{array}$ \\
$\begin{array}{l}\text { Videofluoroscopy and videoendoscopy provide dynamic } \\
\text { imaging of the swallowing functions. The assessment is } \\
\text { aimed at confirming the diagnosis of dysphagia, } \\
\text { characterizing it, and supporting the design of the } \\
\text { intervention plan. }\end{array}$ \\
\hline
\end{tabular}

Table 2 Proposed protocol for screening and intervention in COVID-19 patients.

\begin{tabular}{|c|c|}
\hline \multicolumn{2}{|l|}{ Screening } \\
\hline Assessment & Description \\
\hline MUST [9] & $\begin{array}{l}\text { 1. Low } \mathrm{BMI}^{\mathrm{a}} \\
\text { 2. Weight loss in } 3-6 \text { months }{ }^{\mathrm{b}} \\
\text { 3. Acute disease effect that has induced a phase of nil-per-os for }>5 \text { days }\end{array}$ \\
\hline Reduced muscle mass [10] & $\begin{array}{l}\text { Assessed by DXA or corresponding standards such as BIA, CT or MRI. Mid-arm muscle or calf } \\
\text { circumference can be used as a surrogate diagnostic measures of muscle mass in settings where no other } \\
\text { diagnostic tools are available. }\end{array}$ \\
\hline EAT-10 [8] & $\begin{array}{l}\text { - Weight loss because of swallowing problems } \\
\text { - Difficulties in swallowing/eating }\end{array}$ \\
\hline Kennedy classification for masticatory function [11] & $\begin{array}{l}\text { Partially edentulous arches are divided into four classes: } \\
\text { - Class I: Bilateral edentulous areas located posterior to the remaining natural teeth } \\
\text { - Class II: A unilateral edentulous area located posterior to the remaining natural teeth } \\
\text { - Class III: A unilateral edentulous area with natural teeth located both anterior and posterior to it } \\
\text { - Class IV: A single but bilateral (crossing the midline) edentulous area located anterior to the remaining } \\
\text { natural teeth }\end{array}$ \\
\hline
\end{tabular}

Nutritional interventions

At admission and during hospital stay [10]

Intervention

1 st step

Dietary counseling and/or food modification

\section{Description}

- Energy ${ }^{\mathrm{c}}$ : 27-30 Kcal/Kg of BW/day (particular attention should be paid to RFS)

- Proteins ${ }^{\mathrm{c}}$ :

- At least $1.0 \mathrm{~g} / \mathrm{Kg}$ of BW/day

- $1.2-1.5 \mathrm{~g} / \mathrm{Kg}$ of $\mathrm{BW} /$ day in presence of acute or chronic diseases

- Up to $2.0 \mathrm{~g} / \mathrm{Kg}$ of BW/day in presence of highly catabolic conditions

- Vitamins and minerals: according to daily allowances.

- EEAs supplementation (i.e. 10-15 g with at least $3 \mathrm{~g}$ of leucine) or

2nd step

- Multi-nutrient formulas providing both macro- and micronutrients (at least $400 \mathrm{kcal} /$ day including $30 \mathrm{~g}$ or more of protein/day).

They should be given to older people with or at risk for malnutrition who fail to ingest adequate amounts of energy and nutrients with foods.

3rd step

Enteral $(\mathrm{EN})$ or parenteral $(\mathrm{PN})$ nutrition

- Early NGT tube in COVID-19 patients requiring mechanical ventilation in the ICU;

- Post-pyloric feeding in those with gastric intolerance or at high risk for aspiration. The prone position is considered safe for EN.

- PN should be considered when EN is not indicated or unable to reach targets.

Particular attention should be paid to RFS.

\section{Rehabilitation post COVID-19}

Nutrition care plan

As per 1st, 2nd and 3rd step

Dysphagia rehabilitation

- Compensatory strategies: postural adjustments, swallowing maneuvers, and diet modifications;

- Rehabilitave strategies: head raising exercises and tongue strengthening exercises.

Rehabilitation of masticatory function

Prosthetic rehabilitation and dental restoration as per patient needs.

MUST Malnutrition Universal Screening Tool, EAT-10 Eating Assessment Tool; EAAs: Essential Amino Acids, $B W$ Body Weight, EN Enteral Nutrition, $P N$ Parenteral Nutrition, NGT Nasogastric Tube, RFS Re-feeding Syndrome, ICU Intensive Care Unit.

${ }^{a}$ Refer to self-reported or estimated values if scales and/or stadiometers cannot be used (i.e., unavailability, hygiene reasons, containment measures).

${ }^{\mathrm{b}}$ Consider video conferencing aids to retrieve previous information from relatives or caregivers (i.e., weight loss, reduced dietary intake, swallowing and masticatory difficulties) in patients who are not able to respond.

${ }^{\mathrm{c}}$ These values should be individually adapted to nutritional status, disease status, pre-illness physical activity level and preferences.

dysphagia has been indicated with the concept of "presbyphagia". This latter is not a pathological condition, but occurs with the aging process and determines subtle changes in the swallowing dynamic paving the way for future impaired swallowing [7]. In other words, presbyphagia may predispose to overt dysphagia. The diagnosis of 
swallowing disorders is composed by a three step process (Table 1) [7].

Instrumental evaluations frequently cannot be performed because not readily available in most settings. However, screening instruments for the early identifications of swallowing impairments are available, easy to be implemented, and sometimes may be even used for diagnostic purposes. For example, the EAT-10 [8] tool is a simple, 10-item questionnaire providing an objective evaluation of swallowing difficulties.

Beyond the swallowing function, it is also important to consider the masticatory function. Dental problems may have a critical impact on the nutritional status, especially in older persons. Poor oral health may result in increased risk of malnutrition, sarcopenia, and frailty [6].

The assessment of nutritional status, swallowing capacity, and masticatory function is highly recommended as part of the normal clinical practice. Here we propose a protocol for nutritional screening and interventions in COVID-19 patients (Table 2). However, these critical aspects contributing to the individual's health are often neglected in normal times. It is not surprising that they have been frequently overlooked during the COVID-19 pandemic as soon as the clinical focus has been shifted towards the treatment of the coronavirus infection. The fact that the standard assessment of the nutritional status can be more challenging during the COVID-19 pandemic does not justify such superficial approach, especially in those patients developing the most severe forms of the disease (and thus at risk of wasting syndromes). In this scenario, clinicians may still rely on rapid screening tools able to identify people at risk of malnutrition, swallowing disorders and/or masticatory problems. Direct interviews with the patient about recent dietary patterns or weight changes might be difficult to conduct because of the severe respiratory conditions as well as for the presence of other comorbidities (e.g., cognitive decline, low level of consciousness). There might also be difficulties at retrieving information from caregivers or relatives due to the lockdown and the limited access to the hospitals. In this context, telemedicine may represent a possible solution for both monitoring the patient as well as obtaining additional information from family members.

In conclusion, the nutritional status should be assessed in all patients, especially today in those affected by COVID19. The evaluation should be conducted at the admission and at every major change of the health status. The preliminary data coming from the San Raffaele hospital about malnutrition and dysphagia should foster reflections on the importance of early detecting malnutrition and/or swallowing impairment in order to potentially prevent the most serious consequences of COVID-19. The role of nutrition cannot be any longer overlooked (independently of the SARS-CoV-2 infection), given its relevance for the patients and the healthcare systems.

Author contributions DA and PCP contributed to conceptualizing and writing the manuscript. $\mathrm{MC}$ and $\mathrm{AD}$ edited and revised the manuscript. DA, PCP, AD, and MC approved the final version of manuscript. All authors have read and agreed to the published version of the manuscript.

\section{Compliance with ethical standards}

Conflict of interest The authors declare that they have no conflict of interest.

Publisher's note Springer Nature remains neutral with regard to jurisdictional claims in published maps and institutional affiliations.

\section{References}

1. Brugliera L, Spina A, Castellazzi P, Cimino P, Arcuri P, Negro A, et al. Nutritional management of COVID-19 patients in a rehabilitation unit. Eur J Clin Nutr. 2020;74:860-3.

2. Cruz-Jentoft AJ, Sayer AA. Sarcopenia. Lancet 2019;393:2636-46.

3. Leslie W, Hankey C. Aging, nutritional status and health. Healthc (Basel). 2015;3:648-58.

4. Jung UJ, Choi M-S. Obesity and its metabolic complications: the role of adipokines and the relationship between obesity, inflammation, insulin resistance, dyslipidemia and nonalcoholic fatty liver disease. Int J Mol Sci. 2014;15:6184-223.

5. Schett G, Sticherling M, Neurath MF. COVID-19: risk for cytokine targeting in chronic inflammatory diseases? Nat Rev Immunol. 2020;20:271-2.

6. Azzolino D, Passarelli PC, De Angelis P, Piccirillo GB, D'Addona A, Cesari M. Poor oral health as a determinant of malnutrition and sarcopenia. Nutrients. 2019;11:2898

7. Wakabayashi H. Presbyphagia and sarcopenic dysphagia: association between aging, sarcopenia, and deglutition disorders. J Frailty Aging. 2014;3:97-103.

8. Belafsky PC, Mouadeb DA, Rees CJ, Pryor JC, Postma GN, Allen $\mathrm{J}$, et al. Validity and reliability of the eating assessment tool (EAT10). Ann Otol Rhinol Laryngol. 2008;117:919-24.

9. Stratton RJ, Hackston A, Longmore D, Dixon R, Price S, Stroud $\mathrm{M}$, et al. Malnutrition in hospital outpatients and inpatients: prevalence, concurrent validity and ease of use of the 'malnutrition universal screening tool' ('MUST') for adults. $\mathrm{Br} \mathrm{J}$ Nutr. 2004;92:799-808.

10. Barazzoni R, Bischoff SC, Breda J, Wickramasinghe K, Krznaric $Z$, Nitzan D, et al. ESPEN expert statements and practical guidance for nutritional management of individuals with SARS-CoV2 infection. Clin Nutr. 2020. https://www.ncbi.nlm.nih.gov/pmc/a rticles/PMC7138149/.

11. Şakar O. Classification of partially edentulous arches. In: Şakar O, editor. Removable partial dentures: a practitioners' manual. Cham: Springer International Publishing; 2016. pp. 17-21. https://doi. org/10.1007/978-3-319-20556-4_3. 